\title{
ОЦЕНКА ИНВЕСТИЦИОННЫХ ПРОЕКТОВ МЕТОДОМ РЕАЛЬНЫХ ОПЦИОНОВ В ОТРАСЛИ ТЕЛЕКОММУНИКАЦИЙ
}

\author{
(C) 2018 Вощинин Вадим Валерьевич \\ аспирант, кафедра «Корпоративные финансы и оценка бизнеса» \\ Санкт-Петербургский Государственный Экономический Университет \\ 191023, Санкт-Петербург, Садовая ул., д. 21 \\ E-mail: automat18@yandex.ru
}

В статье проведён краткий анализ особенностей инвестиционных проектов в сфере телекоммуникаций и их оценки методом реальных опционов. Выявлены недостатки наиболее распространенных и применяемых в отечественной практике методов и моделей.

Ключевые слова: отрасль телекоммуникаций; экономика; инновационные проекты, реальные опционы.

В отрасли телекоммуникаций инвестиционная деятельность носит ключевой характер, без нее невозможно развитие бизнеса, удержание доли рынка и развитие новых конкурентных технологий. В настоящее время среди российских компаний наиболее используемыми являются традиционные методы оценки проектов, в основе которых лежит принцип стоимости денег во времени и дисконтирования будущих денежных потоков, генерируемых вложенными инвестициями. В зависимости от целей оценки, в качестве ориентира используются такие показатели как чистая текущая стоимость $(N P V)$ - для оценки эффекта от реализации проекта, срок окупаемости $(D P P)$ - для отсечения проектов, превышающих допустимый срок возврата вложенных средств, а также индекс рентабельности $(P I)$ - для выбора наиболее эффективного проекта из ряда аналогичных при ограниченных ресурсах.

При использовании этих показателей аналитики сталкиваются с рядом ограничений и допущений, присущих методам дисконтирования. В первую очередь, это связано с тем, что большая часть проектов носит инновационный характер, следовательно, имеют повышенную неопределенность в будущем, а при дисконтировании редко учитываются риски конкретного проекта помимо стоимости капитала и общих рисков компании. Вторая причина - отсутствие исторических данных по аналогичным проектам. Вместе с тем, инвестиционные проекты в телекоммуникационной сфере характеризуются значительным объемом первоначальных инвестиций при длительном сроке реализации и долгосрочной окупаемости.

В результате, если анализировать статистику реализации инновационных проектов, то три из четырех заканчиваются неудачей [5].

Ситуация усугубляется продолжающимися экономическими кризисами, как мировыми, так и в нашей стране. Необходимость наращивания прибыли, которую диктуют акционеры, обуславливает экономию затрат, а также детальный анализ инвестиционной деятельности. В этой ситуации топ-менеджмент не готов расширять портфель инвестиционных проектов и вкладывать средства в рискованные мероприятия или активы. Это приводит к снижению конкурентного преимущества, отсутствию инновационных решений и разработок.

Интересным инструментом для анализа инвестиционных проектов в сфере телекоммуникаций является метод реальных опционов (Real Option Valuation, ROV), который используется в отраслях с высокой зависимостью от изменений на рынке, в том числе в нефтедобывающей и нефтеперерабатывающей отраслях, банковской сфере либо деятельности на фондовых и сырьевых биржах. В российской практике опыт применения реальных опционов имеют крупнейшие нефтедобывающие компании («Газпром»), а также апробация на отдельных проектах методов реальных опционов проводилась в компании МТС.

При этом широкое применение данного метода в настоящее время находится в процессе становления. Разрыв между применяемыми методами оценки проектов и потребностями бизнеса подчеркивает актуальность исследования метода реальных и разработки практических методик оценки. Необходимо внедрение в 
бизнес новых методов, отражающих изменчивость рынка, оперативное принятие управленческих решений, а также пользующихся доверием у инвесторов.

K инновационным проектам не применимы рыночные методы оценки, поэтому телекоммуникационные компании и используют доходные методы оценки путем дисконтирования денежных потоков. Этот расчет имеет ряд узких мест:

1) данные методы не учитывают возможность принятия управленческих решений и управленческую гибкость: решения, принятые менеджментом после начала реализации проекта, не могут быть оцифрованы и учтены в момент оценки и принятия решения о старте проекта, а зачастую менеджмент может оказать серьезное влияние на проект, особенно инновационный - поддающийся изменчивости и зависящий от конъюнктуры рынка;

2) оценка риска носит поверхностный характер и учтена в ставке дисконтирования, при этом нет утвержденной методики оценки риска, его учета - каждая компания должна разработать свой собственный подход. Часто в качестве ставки дисконтирования используется показатель средневзвешенной стоимости капитала компании (Weighted Average Cost of Capital, WACC), который отражает ценность денежных средств компании, но не учитывает риски конкретного проекта.

Наиболее удачным с теоретической стороны методом оценки инновационных инвестиционных проектов является ROV, поскольку он позволяет учесть риск конкретного инвестиционного проекта, а также проанализировать возможные сценарии его реализации, заранее сделав поправки на возможные изменения конъюнктуры рынка и принятие управленческих решений (в том числе, оценив их качество в виде отдельного риска).

В отечественной литературе наиболее распространённым и хорошо описанным методом оценки стоимости опциона, применяемом в ROV, является метод, основанный на модели Блэка-Шоулза (Black-Scholes Option Pricing Model). Модель для реальных опционов совпадает с финансовой по количеству компонентов, однако некоторые из них несут иной смысл по сравнению с моделью для оценки финансовых опционов.

$$
\begin{aligned}
& \mathrm{C}_{\text {option }}=\mathrm{PN}\left(\mathrm{d}_{1}\right)-\mathrm{se}^{-\mathrm{rt}} \mathrm{N}\left(\mathrm{d}_{2}\right), \\
& d_{1}=\frac{\ln \frac{p}{s}+\left(r+\frac{v^{2}}{2}\right) t}{v \sqrt{t}} \\
& d_{2}=d_{1}-v \sqrt{t}
\end{aligned}
$$

где C - стоимость опциона, P - ожидаемый денежный поток, $\mathrm{s}-$ расходы на приобретение фондов проекта, $\mathrm{t}$ - период времени от текущего момента до начала реализации проекта, $\mathrm{r}$ - стоимость денег во времени, дисконт, $\mathrm{v}$ - неопределенность, вариация доходности вложений, е число Эйлера, $\mathrm{N}(\mathrm{d})$ - нормальная кумулятивная функция плотности вероятности [2].

В отечественной литературе часто встречается некорректная интерпретация символьных обозначений, относящаяся к модели оценки финансовых, а не реальных опционов.

Проанализировав применение данной модели на практике при оценке инвестиционных проектов, было установлено, что существует ряд ограничений для ее успешного использования в оценке телекоммуникационных проектов:

1) необходимо рассчитать вариацию доходности вложений, то есть изменчивость цены базового актива. В части инвестиционных проектов таким активом выступает показатель NPV. Однако для определения волатильности данного показателя необходимо обладать базой статистических данных по аналогичным проектам, что невозможно, если проект реализуется впервые и носит инновационный характер. Экспертную же оценку данного показателя невозможно достоверно обосновать;

2) серьезным допущением модели является непрерывность времени, которая подразумевается исходя из финансовых опционов, лежащих в основе данного метода. То есть, продать или купить опцион возможно в любой момент времени. Это в корне противоречит модели работы инвестиционных проектов, в которых первоначальные инвестиции вкладываются безвозвратно и отсутствует возможность оперативного выхода из проекта. Кроме того, не применим биржевой принцип - среди инновационных проектов и разработок отсутствует достаточный объем спроса и предложения для оперативной продажи результатов инвестиционной деятельности.

В данной ситуации интересен менее распространённый метод, основанный на модели 
биномиального дерева Кокса-Росса-Рубинштейна (англ. Binomial Option Pricing Model). Оценка стоимости опциона прямо пропорциональна дисконтированной возможной цене и обратно пропорциональна безрисковой ставке. При большом количестве «ветвей» биномиального дерева распределение стремится к нормальному, а результаты, полученные с помощью данного метода, стремятся к оценке по модели Блэка-Шоулза.

Модель в общем виде можно сформулировать следующим образом:

$$
\begin{aligned}
& \mathrm{C}=\frac{P C_{u}+(1-P) C_{d}}{r} \\
& P \equiv \frac{r-d}{u-d}
\end{aligned}
$$

где $\mathrm{C}$ - стоимость call опциона, $\mathrm{C}_{\mathrm{u}}-$ стоимость опциона в случае роста денежного потока, $\mathrm{C}_{\mathrm{d}}$ - стоимость опциона в случае уменьшения денежного потока, d и u - параметры изменения денежного потока, $\mathrm{r}$ - стоимость денег во времени, безрисковая ставка [4].

Таким образом, модель предполагает наличие двух вариантов развития событий на каждом этапе реализации проекта, то есть результаты будут точнее при большем количестве этапов принятия ключевых решений, а также нескольких источниках неопределенности в проекте. И оценка эффекта от проекта представляет собой совокупность вероятностей всех возможных ветвей реализации проекта, и, соответственно, всех решений менеджмента, приведенных к текущей стоимости.

Однако, на практике выявлено, что приме- нение данного метода существенно ограничено биномиальным выбором - доступно только 2 решения на каждом этапе. Данное ограничение допустимо для финансовых опционов, либо простых инвестиционных проектов с несколькими однозначно определенными вариантами решений. Но, ввиду того, что «деревья» инвестиционных проектов в сфере телекоммуникаций имеют сложную структуру, часто несимметричную, значительно распределенную по времени и с большой вариативностью принимаемых решений, целесообразен вывод о том, что два наиболее используемых в отечественной практике метода оценки инвестиционных проектов с помощью реальных опционов имеют ряд существенных допущений и ограничений, которые ставят под сомнение целесообразность применения данных методов для оценки инновационных проектов в сфере телекоммуникаций.

Требуется популяризация, теоретическое обоснование и расширение практического применения (в том числе апробация в российских телекоммуникационных компаниях) новых методов, в которых:

1) входные параметры будут определяться на основании всей доступной информации при планировании и оценке проекта, которая будет полной, понятной и объективной;

2) в основе будут заложены индивидуальные риски, присущие конкретному проекту, а также будет учтена вариативность управленческих решений на этапах реализации проекта;

3) будет возможно построение произвольного дерева управленческих решений, наилучшим образом подходящего для конкретного инвестиционного проекта.

\section{Библиографический список}

1. Круковский А.А. Метод реальных опционов в управлении инвестициями // Труды ИСА РАН. 2008. Т. 37. С. 122-144

2. Black F., Scholes M. The pricing of options and corporate liabilities // Journal of Political Economy. 1973. Vol. 81. P. 637-654.

3. Cox J.C., Ross S.A. The valuation of options for alternative stochastic processes // Journal of Financial Economics. 1976. Vol. 3. P. 145-166.

4. Cox J.C., Ross R.A., Rubinstein M. Option pricing a simplified approach // Journal of Financial Economics. 1976. Vol. 7. P. 229-263.

5. Gage D. The Venture Capital Secret: 3 Out of 4 Start-Ups Fail // The Wall Street Journal. 2012. 